الخصائص الميكانيكية للخرسانة عالية المقاومة المسلحة بالالياف الفولاذية

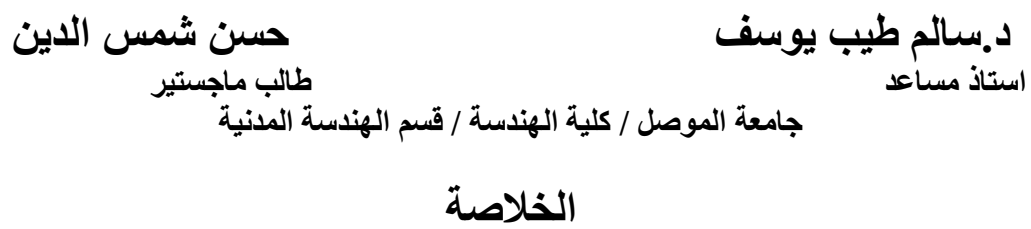

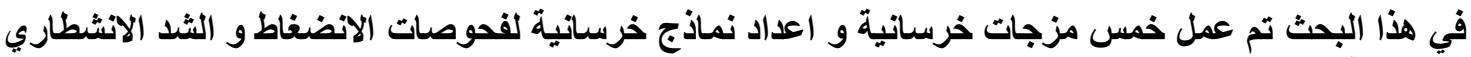

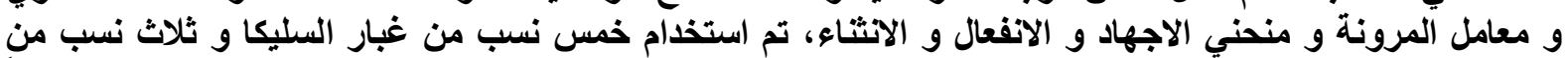

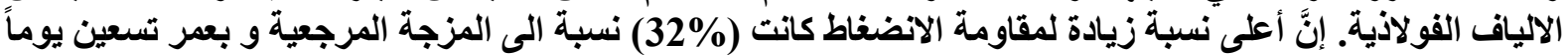

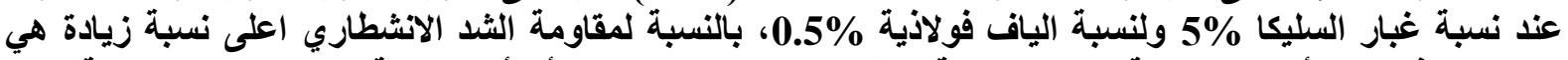

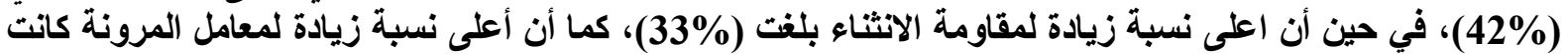

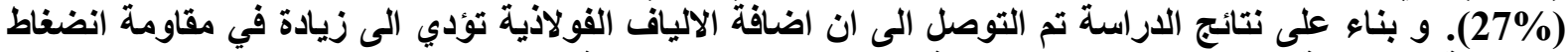

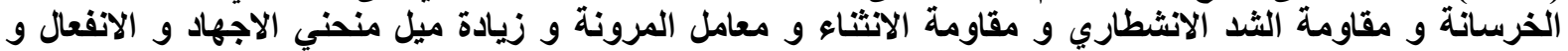

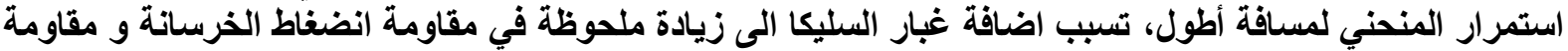
الثد الانثطاري و معامل المرونة و زيادة ميل منحني الاجهاد و الاتفعال، بينما تقلّل من مقاومة الانثناء.

الكلمات الدالة: خرسانة عالية المقاومة، الالياف الفولاذية، غبار السليكا، الخصائص الميكانيكية

\title{
Mechanical Properties of High Strength Steel Fiber Reinforced Concrete
}

\author{
Dr. salim T. Yousif \\ Assistant Professor \\ hisaan Shams-aldeen \\ MSc Student \\ University of Mosul/College of Engineering
}

\begin{abstract}
In this research, five concrete mixes were prepared, made samples for compressive, Splitting, bending and modulus of elasticity test have been use five of silica fume percentages and three of steel fiber percentages. According to results of this study, addition of steel fiber cause increase in compressive strength, splitting strength, bending strength, modulus of elasticity, and increase the angle of stress-strain curve. Addition of silica fume cause increase in compressive strength, splitting strength, modulus of elasticity, increase the angle of stress-strain curve while cause decreasing in bending strength. The higher compressive strength percentage was $(32 \%)$ as compared to reference sample prepared at ninety days with silica fume percentage $(5 \%)$ and steel fiber $(\mathbf{0 . 5 \%})$, The higher percentage for splitting strength was $(\mathbf{4 2 \%})$ while the highest percentage for modulus of rupture $(33 \%)$, The highest ratio for modulus of elasticity $(27 \%)$.
\end{abstract}

Keywords: High strength concrete, Steel fibers, Silica fume, mechanical properties 


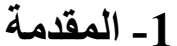

نتيجة لتز ايد المتطلبات التكنولوجية و المعمارية فقد أوجدت أنواع جديدة من الخرسانة من أهمها الخرسانة عالية

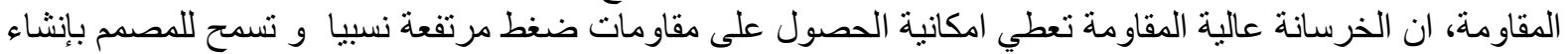

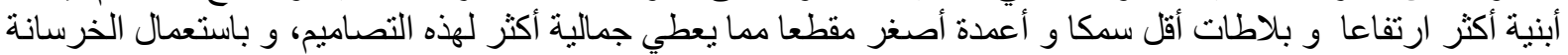

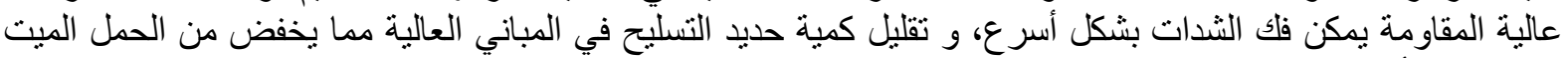

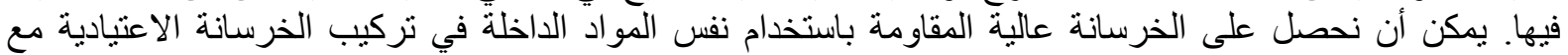

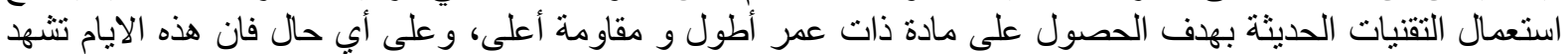

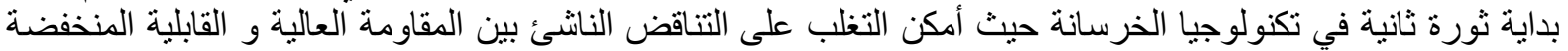

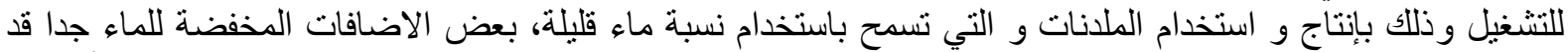

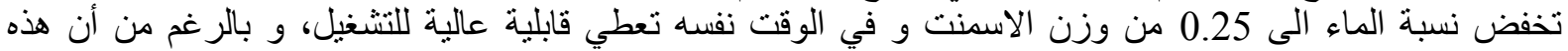

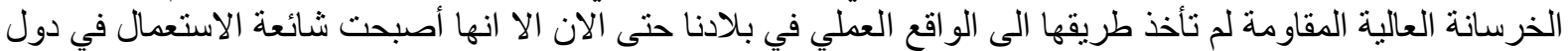

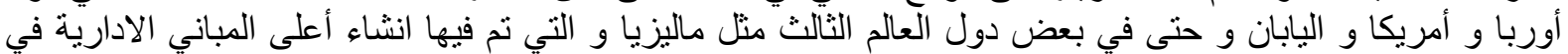

العالم.

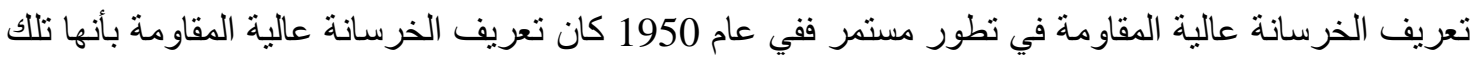

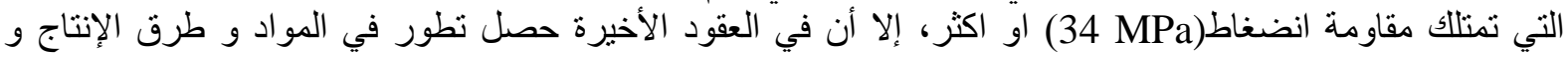

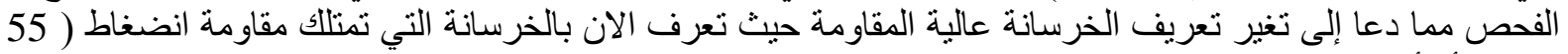

(MPa

أجرى الباحث (Samman) وأخرون [2] في عام الجام (1999) دراسة عن الخصائص الميكانيكية للخرسانة

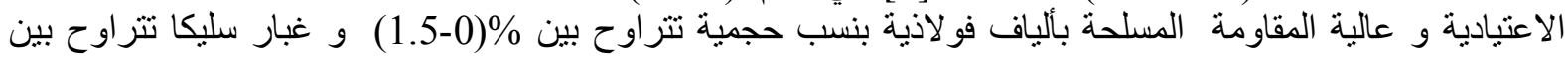

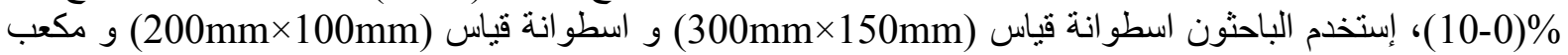

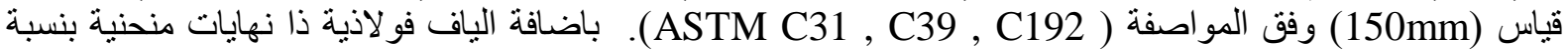

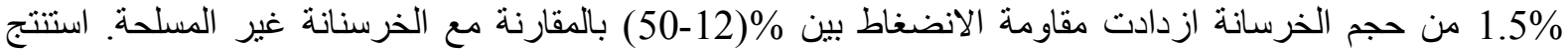

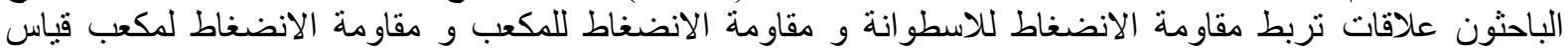

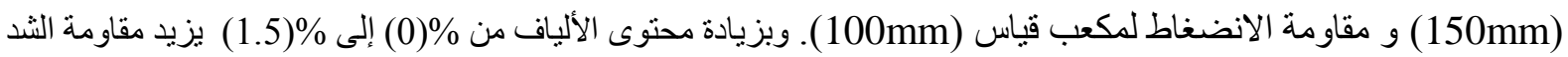
الانشطاري بحلود 150) 53\% إلى

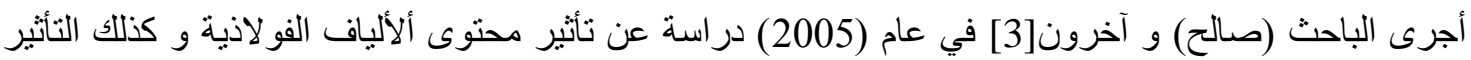

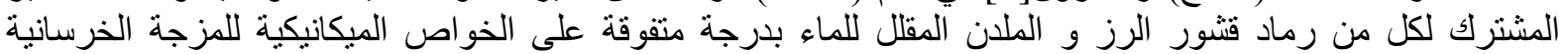

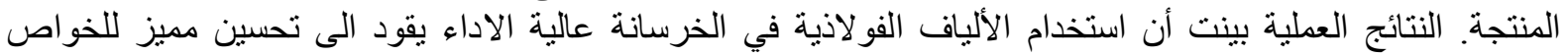
الميكانيكية للخرسانة النجانة

قام الباحث ( Katkhuda) و أخرون [4] عام (2009) بدراسة عملية حول تاثير غبار السليكا على الخصائص

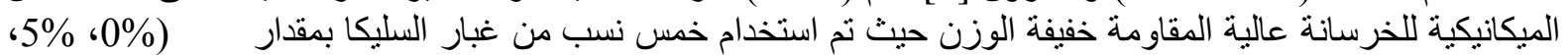

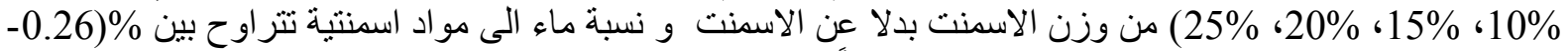

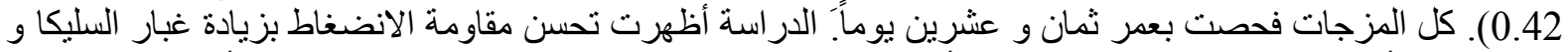

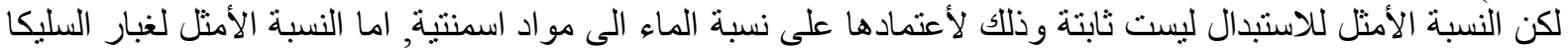

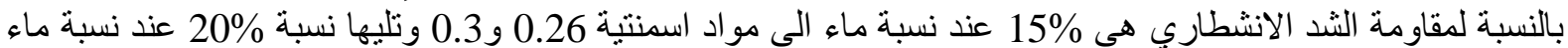

$$
\text { الى مواد اسمنتية } 0.34 \text { و } 0.38 \text { و } 0.42 .
$$

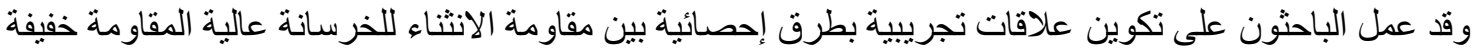

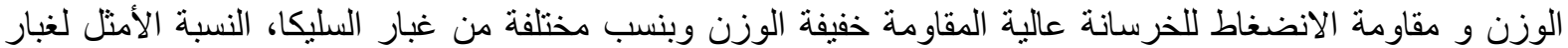

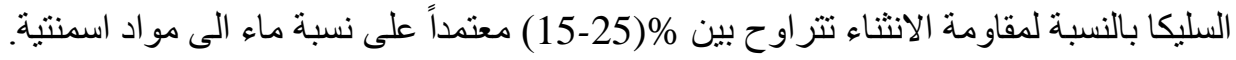

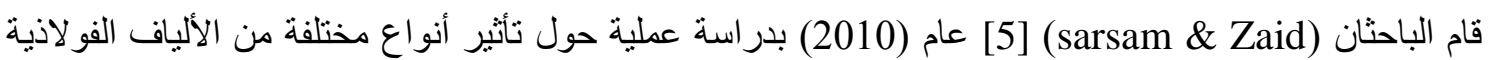
وبنسب حجميه مختلفة تتراوح بين صفر إلى 2\% على على الخرسانة، تراوحت مقاومة الخرسانة من

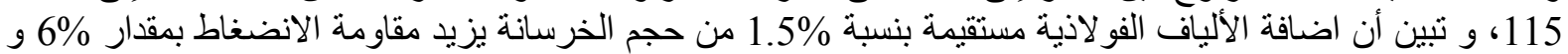
زيادة بمقدار 17.5\% بالنسبة للإضافة 1.5\% ألياف فو لاذية منحنية النهايات، و عموما بوجود الألياف تختلف خواصنة الخرسانة بشكل واضح عن خو اص الخرسانة غير المسلحة. 
عند اضافة الالياف الفو لاذية المستقيمة بنسبة 1.5\% من حجم الخرسانة ازدادت مقاومة الانثناء بمقدار $24.5 \%$ و و

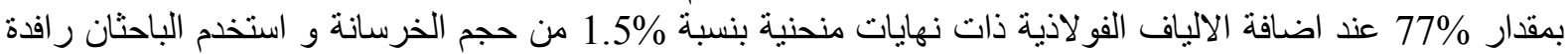
. ASTM C78 بقأبعاد المو اصفة (400mm×100mm×100mm (prism)

كما تمت دراسة تأثثر الألياف الفو لاذية على مقاومة الثد الانشطاري، حيث عند اضافة الالياف الفو لاذية المستقيمة

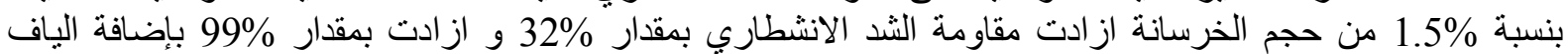

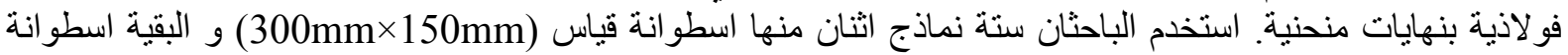
قياس (200mm×100mm) وفق المو اصفة (ASTM C496).

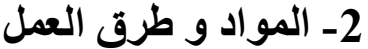

تم استخدام المواد المتوفرة محليا و المتمثلة بالماء، الركام الناعم، الركام الخشن و الاسمنت و المضافات و ذلك بعد مقارنتها مع المو اصفات و بالثنكل التالي: 1-2 :الاسمنت: استخدم في هذا البحث سمنت ماردين نركي المنشأ منوفر محليا. يبين الجدول (1) نتائج التحليل الكيمبائي للإسمنت، أما الجدول (2) فيبين الخصائص الفيزيائية للإسمنت و تم الفحص وفق المواصفة العر اقية ,

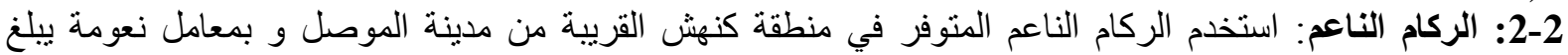

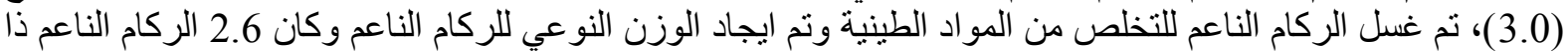

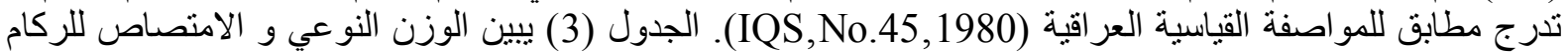

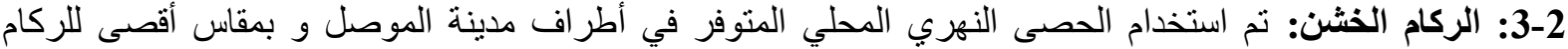

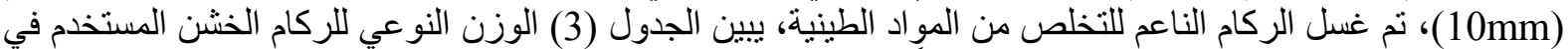

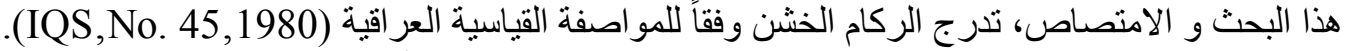
الجدول (1) الخصائص الكيميائية للسمنت

\begin{tabular}{|c|c|c|c|c|c|}
\hline $\begin{array}{c}\text { Chemical } \\
\text { Composition }\end{array}$ & Value \% & Limits \% & $\begin{array}{c}\text { Chemical } \\
\text { Components }\end{array}$ & Value \% & Limits \% \\
\hline $\mathrm{SiO}_{2}$ & 20.8 & --- & $\mathrm{C}_{3} \mathrm{~S}$ & 57.06 & --- \\
\hline $\mathrm{AL}_{2} \mathrm{O}_{3}$ & 4.28 & --- & $\mathrm{C}_{2} \mathrm{~S}$ & 16.59 & --- \\
\hline $\mathrm{Fe}_{2} \mathrm{O}_{3}$ & 5.00 & --- & $\mathrm{C}_{3} \mathrm{~A}$ & 2.89 & 5 \\
\hline $\mathrm{CaO}$ & 62.32 & --- & $\mathrm{C}_{4} \mathrm{AF}$ & 15.22 & --- \\
\hline $\mathrm{MgO}$ & 2.95 & 5 & L.S.F. & 0.916 & --- \\
\hline $\mathrm{SO}_{3}$ & 1.78 & 2.3 & Solid solution & 17.50 & --- \\
\hline Free Lime & 1.18 & --- & & & --- \\
\hline Loss on ignition & 2.83 & 3 & & & --- \\
\hline Insoluble residue & 0.75 & 1.5 & & & --- \\
\hline Total & 99.97 & --- & & & --- \\
\hline
\end{tabular}

الجدول (2) الخصائص الفيزيائية للسمنت

\begin{tabular}{|c|c|c|}
\hline Physical properties & $\begin{array}{c}\text { Test } \\
\text { results }\end{array}$ & $\begin{array}{c}\text { Limits of Iraqi Specification } \\
\text { NO.5/1984 }\end{array}$ \\
\hline $\begin{array}{c}\text { Setting time (vicat's apparatus) } \\
\text { Initial setting time, hrs: min. }\end{array}$ & $1: 20$ & $\geq 1 \mathrm{hr}$ \\
Final setting time, hrs: min. & $2: 40$ & $\leq 10 \mathrm{hrs}$ \\
\hline Fineness & $2 \%$ & $\leq 10 \%$ \\
\hline Compressive strength & 26.6 & $\geq 15$ \\
3days, N/mm & 33.2 & $\geq 23$ \\
\hline 7days, N/mm & & \\
\hline
\end{tabular}


الجدول (3) الوزن النوعي و الامتصاص للركام الناعم و الخثن

\begin{tabular}{|c|c|c|}
\hline \multirow{2}{*}{ Physical properties } & \multicolumn{2}{|c|}{ Test results } \\
\cline { 2 - 3 } & Sand & Gravel \\
\hline Specific gravity & 2.60 & 2.63 \\
\hline Absorption & $1 \%$ & $1 \%$ \\
\hline Bulk density & $1850 \mathrm{~kg} \backslash \mathrm{m}^{3}$ & $1650 \mathrm{~kg} \backslash \mathrm{m}^{3}$ \\
\hline
\end{tabular}

4-2: الماء : استخدم ماء الثرب الاعتيادي في مدينة الموصل لجميع المزجات الخرسانية المستخدمة في هذا البحث. 5-2: الملان الفائق (Superplasticizer ): في هذا البحث استخدم ملان باسم (P) (PV)-H (modified) لتقليل ماء

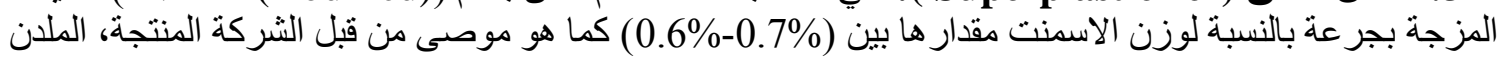
يحسن خصائص الخرسانة.

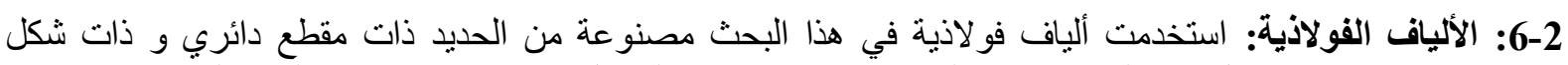

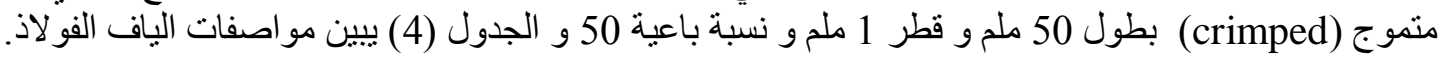
الجدول (4) مواصفات الألياف الفولاذية

\begin{tabular}{|c|c|c|c|c|}
\hline $\begin{array}{c}\text { Fiber } \\
\text { type }\end{array}$ & $\begin{array}{c}\text { Tensile strength } \\
(\mathbf{M P a})\end{array}$ & $\begin{array}{c}\text { Young modulus } \\
(\mathbf{G P a})\end{array}$ & $\begin{array}{c}\text { Ultimate } \\
\text { Elongation percent }\end{array}$ & $\begin{array}{c}\text { Specific } \\
\text { Gravity }\end{array}$ \\
\hline steel & $>1000$ & 200 & $30-25$ & 7.85 \\
\hline
\end{tabular}

7-2 :غبار السليكا: هي مادة تتكون من حبيبات دقيقة جداً مساحتها السطحية حو الى أربعة إلى خمسة أمثال المساحة

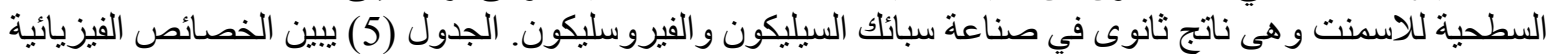
لغبار السليكا.

الجدول (5) الخصائص الفيزيائية لغبار السليكا مع المواصفة ASTM C1240-03

\begin{tabular}{|c|c|c|}
\hline Physical properties & Standard limits & SF \\
\hline Specific surface area, $\min ,\left(\mathrm{m}^{2} / \mathrm{g}\right)$ & 15 & 20 \\
\hline $\begin{array}{c}\text { Strength activity Index with Portland cement at } \\
\text { 7days, min. percent of control. }\end{array}$ & 105 & 196 \\
\hline Percent retained on $45 \mu \mathrm{m}($ No.325), max, \% & 10 & 7 \\
\hline
\end{tabular}

صمدت المزجة الخرسانية عالية المقاومة بالاعتماد على المواصفة (ACI 211.4R-08 ) [6] و و الجدول (6) يبين

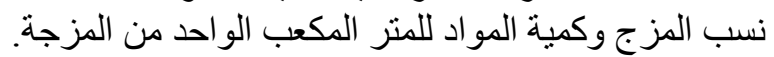
في هذا البحث تم اجراء الفحوصات المبينة أدناه للحصول على على الخصائص الميكانيكية للخرسانة عالية المقاومة وحسب المواصفات المبينة مع كل فحص:

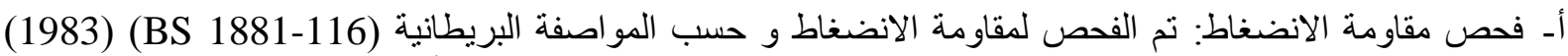

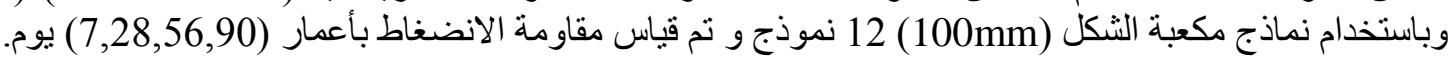
بـ فحص الثد الانشطاري: تم فحص مقاومة الثد الانشطاري و والنماذج المعدة للفحص اسطوانة بأبعاد (300mm×150mm)

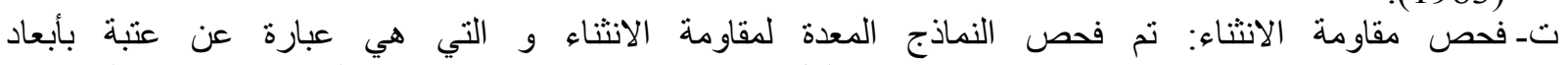
(500mm×100mm×100mm) .(1983) (BS 1881-118)

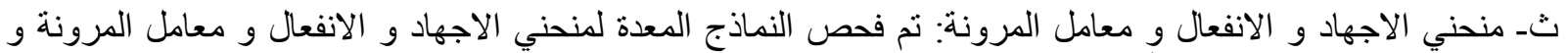

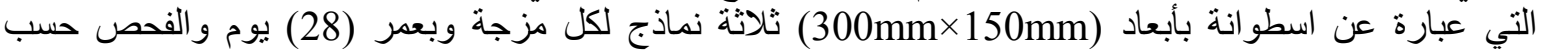


المواصفة (ASTM C 469-94) و باستخدام جهاز (Compressometer) و و جهاز (Dial gague) و جهاز لتسليط ضغط (ALPH) و تم بعد ذللك رسم منحني الإجهاد و الانفعال و حساب معامل المرونة لكل المزجات الخرسانية.

$$
\text { الجدول (6) نسب المواد للمزجة المرجعية }
$$

\begin{tabular}{|c|c|c|c|c|c|}
\hline Weight percent\% & $\begin{array}{c}\text { Superplasticier } \\
\mathbf{L}_{\mathbf{M}} \mathbf{M}^{\mathbf{3}}\end{array}$ & $\begin{array}{c}\text { Water } \\
\mathbf{K g} / \mathbf{m}^{\mathbf{3}}\end{array}$ & $\begin{array}{c}\text { Sand } \\
\mathbf{K g} / \mathbf{m}^{\mathbf{3}}\end{array}$ & $\begin{array}{c}\text { Coarse } \\
\mathbf{K g} / \mathbf{m}^{\mathbf{3}}\end{array}$ & $\begin{array}{c}\text { Cement } \\
\mathbf{K g} / \mathbf{m}^{\mathbf{3}}\end{array}$ \\
\hline 1:1.11:1.69:0.285:0.008 & 4.74 & 169 & 658 & 1000 & 593 \\
\hline
\end{tabular}

\section{3-3 - 3 النتائج و المناقشة \\ 1-3 - 1 مقاومة الانضغاط}

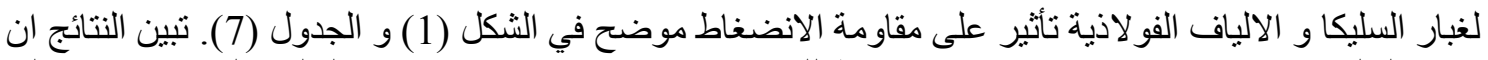

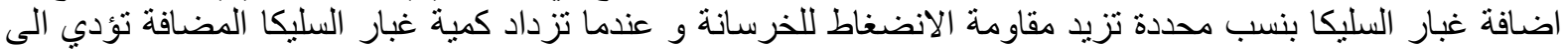

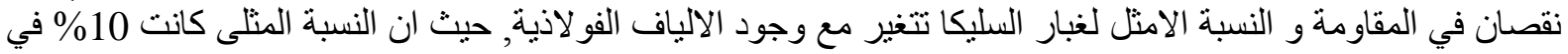

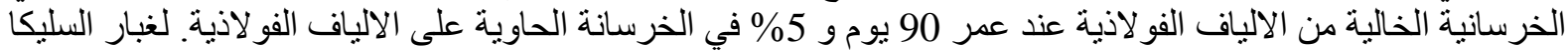

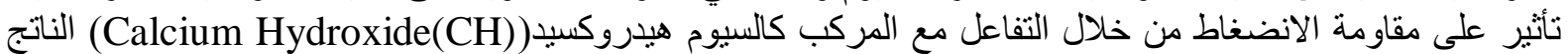

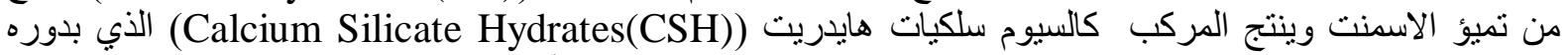

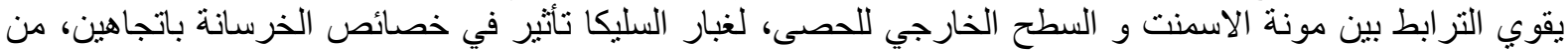

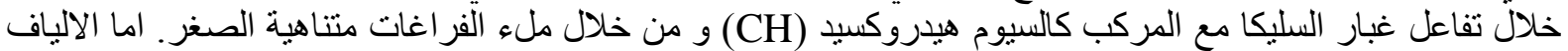

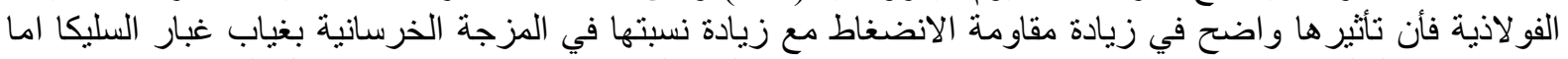

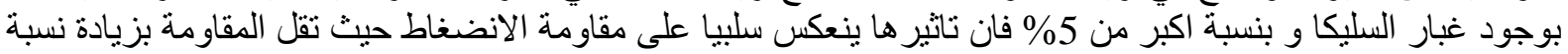

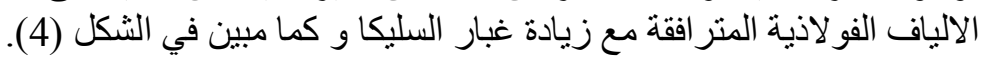

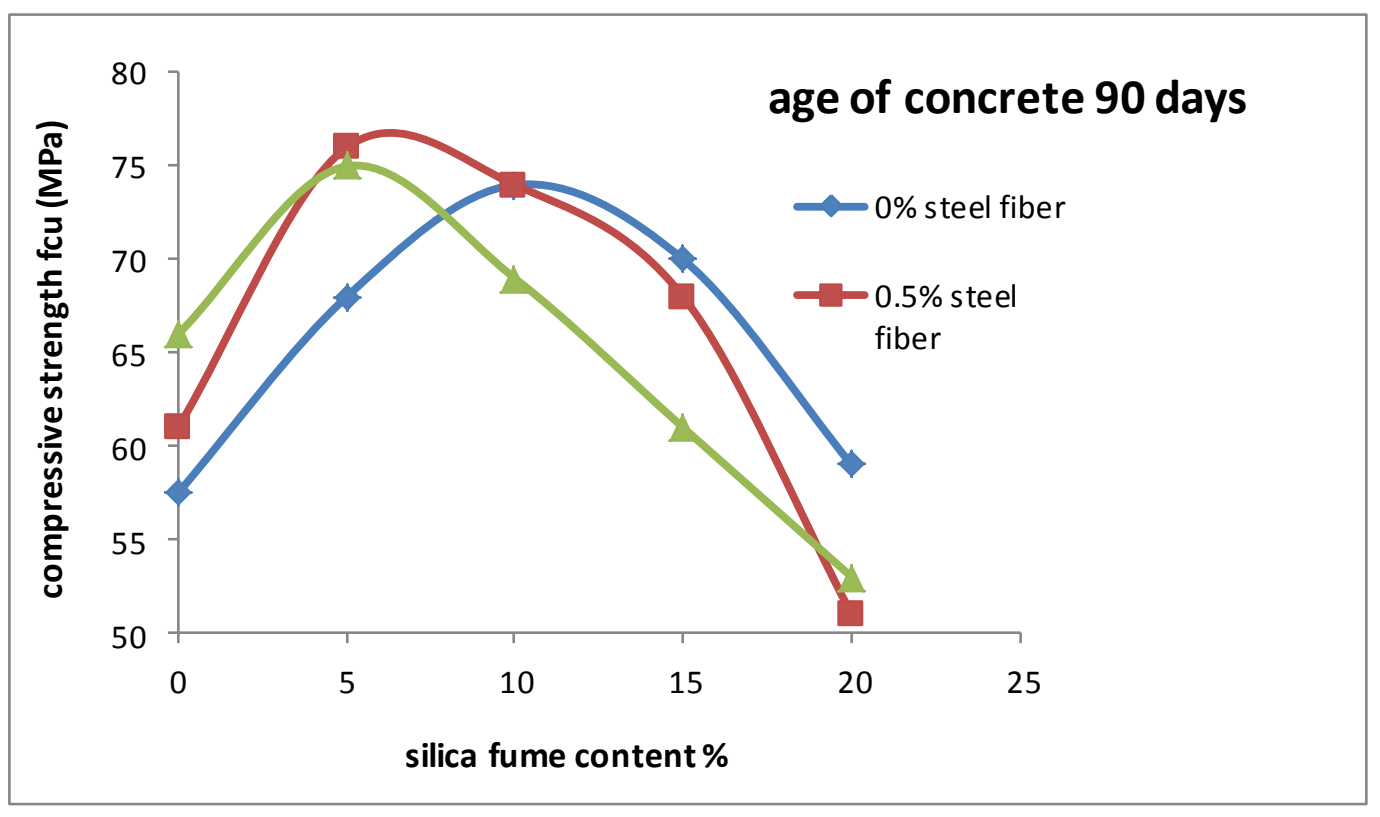

الشكل (1) تأثير غبار السليكا على مقاومة انضغاط الخرسانة 


\section{الجدول (7) قيم مقاومة الانضغاط للمزجات الخرسانية}

\begin{tabular}{|c|c|c|c|c|c|c|}
\hline Mix & SF \% by & Steel fiber \% & \multicolumn{4}{|c|}{ Compressive Strength (MPa) } \\
\cline { 4 - 7 } designation & $\begin{array}{c}\text { weight of } \\
\text { cement }\end{array}$ & $\begin{array}{c}\text { by volume of } \\
\text { concrete }\end{array}$ & $\begin{array}{c}\text { 7 } \\
\text { Days } \\
\text { (S7 d) }\end{array}$ & $\begin{array}{c}\mathbf{2 8} \\
\text { Days } \\
\text { (S28 d) }\end{array}$ & $\begin{array}{c}\mathbf{5 6} \\
\text { Days } \\
\text { (S56 d) }\end{array}$ & $\begin{array}{c}\text { 90 } \\
\text { Days } \\
\text { (S90 d) }\end{array}$ \\
\hline M 11 & 0 & 0.0 & 36.5 & 45 & 52.0 & 57.5 \\
\hline M 12 & 0 & 0.5 & 39.8 & 48 & 54.8 & 61.0 \\
\hline M 13 & 0 & 1.0 & 47.0 & 62 & 65.0 & 66.0 \\
\hline M 21 & 5 & 0.0 & 42.0 & 64 & 65.0 & 68.0 \\
\hline M 22 & 5 & 0.5 & 50.0 & 60 & 66.0 & 76.0 \\
\hline M 23 & 5 & 1.0 & 48.0 & 57 & 65.0 & 75.0 \\
\hline M 31 & 10 & 0.0 & 46.0 & 56 & 66.0 & 74.0 \\
\hline M 32 & 10 & 0.5 & 44.0 & 55 & 64.0 & 74.0 \\
\hline M 33 & 10 & 1.0 & 48.0 & 55 & 60.0 & 69.0 \\
\hline M 41 & 15 & 0.0 & 45.0 & 55 & 66.0 & 70.0 \\
\hline M 42 & 15 & 0.5 & 47.0 & 57 & 63.0 & 68.0 \\
\hline M 43 & 15 & 1.0 & 32.0 & 47 & 59.0 & 61.0 \\
\hline M 51 & 20 & 0.0 & 39.0 & 47 & 56.0 & 59.0 \\
\hline M 52 & 20 & 0.5 & 41.0 & 47 & 49.0 & 51.0 \\
\hline M 53 & 20 & 1.0 & 38.0 & 48 & 50.0 & 53.0 \\
\hline
\end{tabular}

تم تمثيل العلاقة بين مقاومة الانضغاط للنموذج المكعب (100mm) مع مقاومة الانضغاط للنموذج الاسطواني بابعاد (300mm×150mm) بالمعادلة الآتية:

$$
f_{\mathrm{cu}}=1.226 f_{\mathrm{c}}
$$

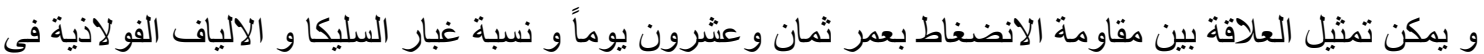
المزجة الخرسانية و مقاومة الانضغاط للمزجة المرجعية بعمر ثمان و عشرين يوماً بالمعادلة بالة الآتية:

$f_{c}=f c r+938.24 v_{f}+33.7 w_{a i}-7741.6 v_{f} w_{a i}$

\section{2-3 2-3 مقاومة الانثناء}

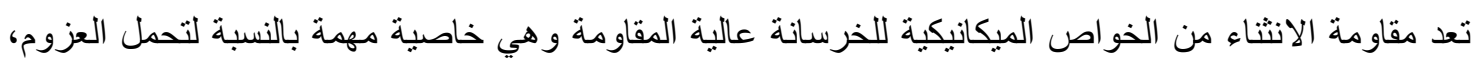

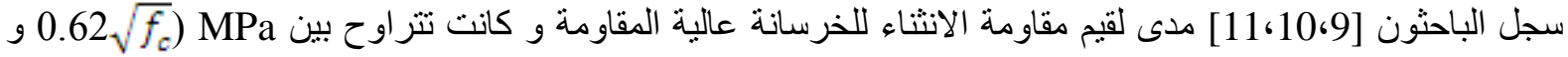

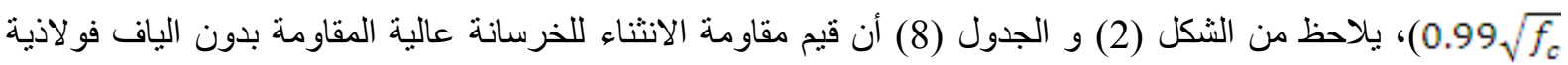

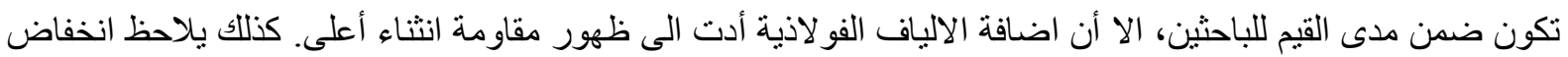

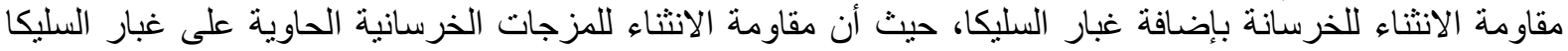

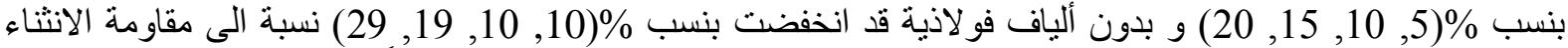

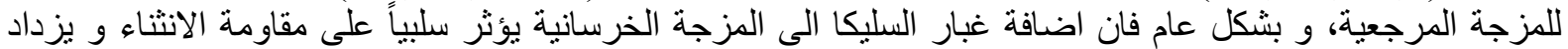

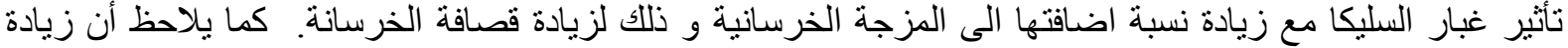

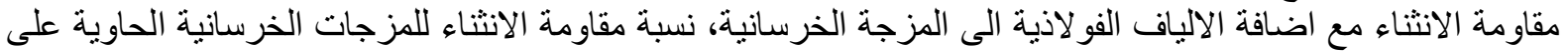

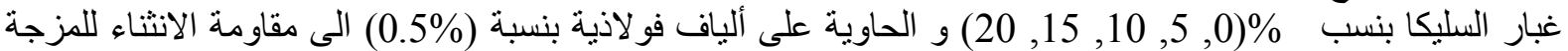

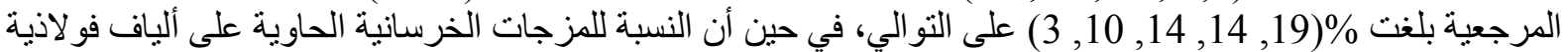

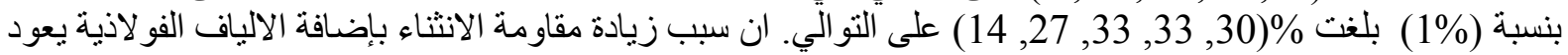

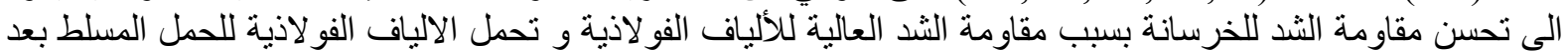

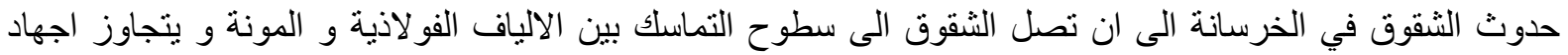


القص مقاومة التماسك العظمى عندئذ يحدث الفثل، علما أن الفثل في الانثناء يحدث خلال الخرسانة و يسبب انفصال

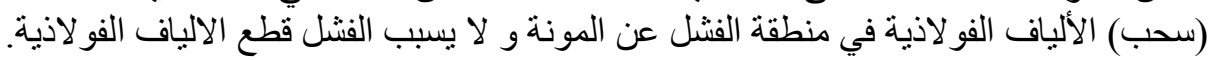

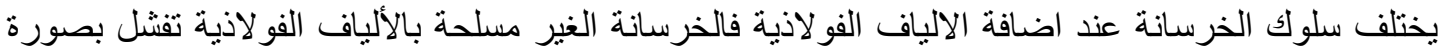

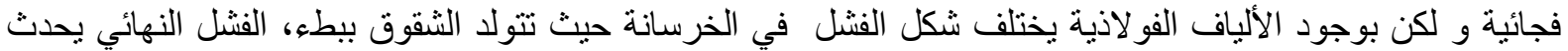

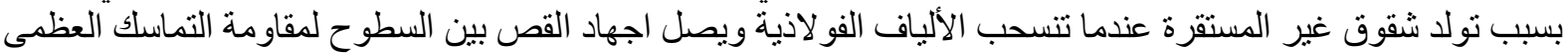

تم تمثيل العلاقة بين مقاومة الانثناء للمزجة الخرسانية و مقاومة الانثناء للمزجة المرجعية و نسب الالياف الفو لاذية و نسب

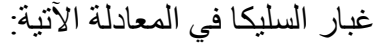

$f_{r}=f_{r T}+250 v_{\mathrm{f}}-7.4 \mathrm{w}_{\mathrm{gi}}$

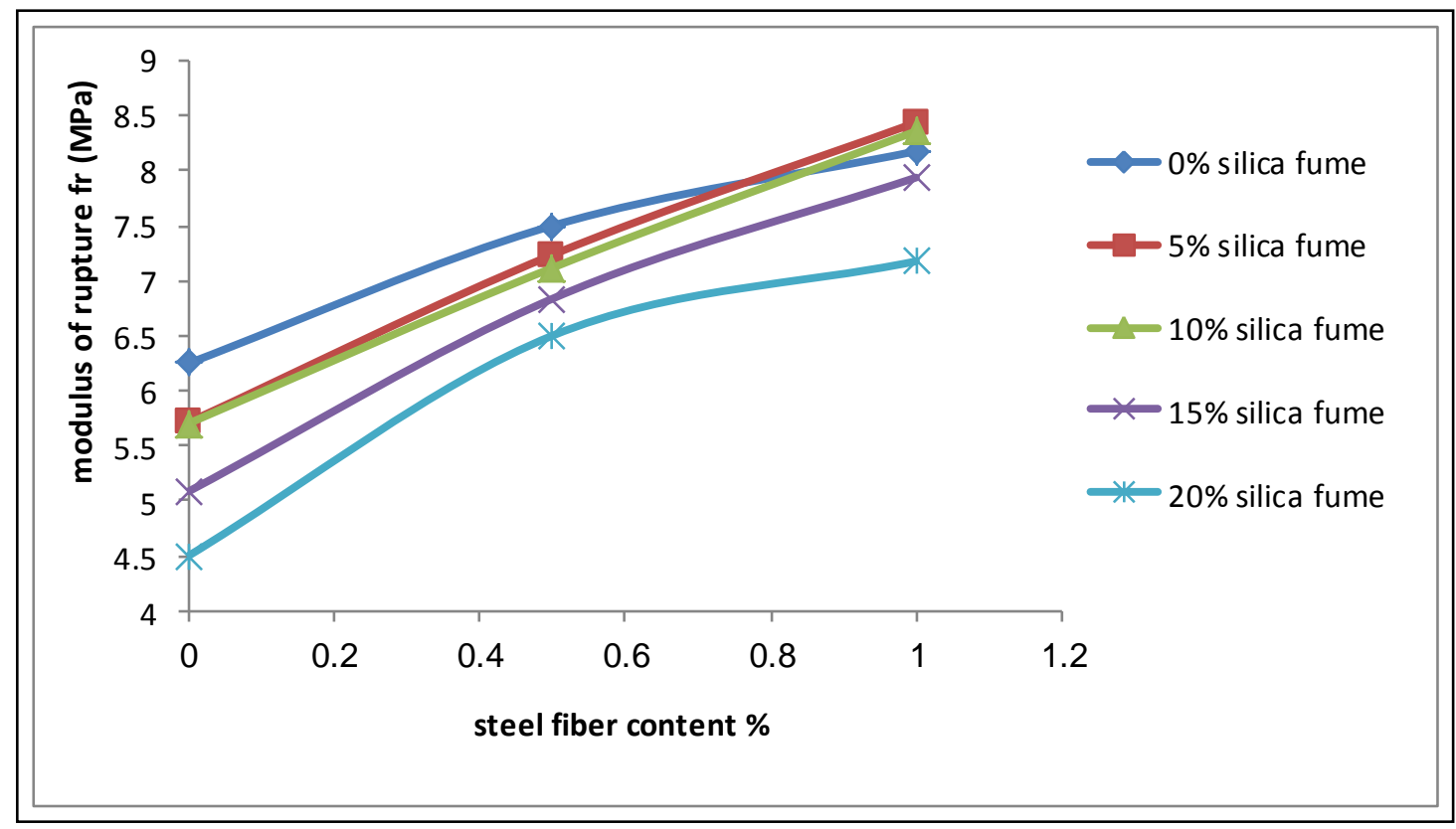

الثكل (2) تغير مقاومة الانثناء مع محتوى ألياف الفولاذ

3-3 -3قاومة الثد الانشطاري

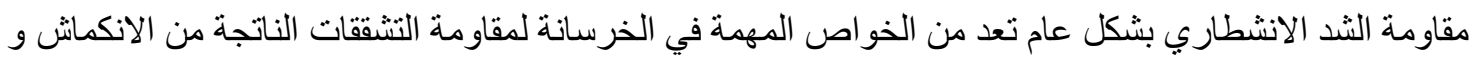

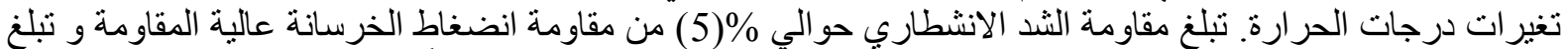

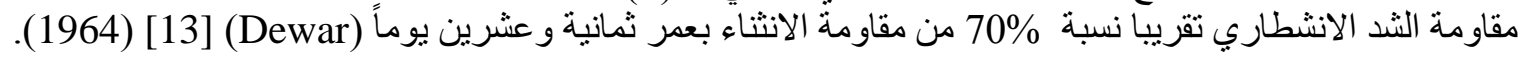
الجدول (9) يبين قيم مقاومة الثد الانتطاري للمزجات الخرسانية وتأثير غبار السليكا و الالياف الفو لاذية عليها.

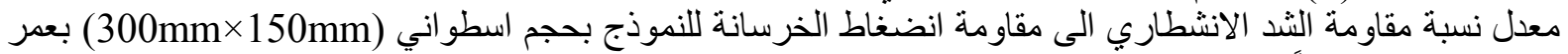

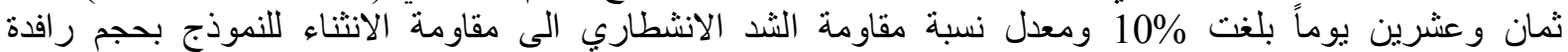
(65\% بلغت $100 \mathrm{~mm} \times 100 \mathrm{~mm} \times 100 \mathrm{~mm})$ 
الجدول (8) قيم مقاومة الانثناء

\begin{tabular}{|c|c|c|c|c|}
\hline Mix Designation & $\begin{array}{l}\text { Modulus of } \\
\text { Rupture } \\
(\text { MPa })\left(f_{r}\right)\end{array}$ & $\begin{array}{c}0.62 \sqrt{f_{c}} \\
\text { Eq ACI 318-05 } \\
\text { (MPa) }\end{array}$ & $\begin{array}{c}0.99 \sqrt{f_{c}} \\
(\mathrm{MPa})\end{array}$ & $\left(f_{r} / f_{r r}\right)$ \\
\hline $\mathrm{M} 11=f_{r T}$ & 6.3 & 4.0 & 6.0 & 1.00 \\
\hline M 12 & 7.5 & 4.1 & 6.2 & 1.19 \\
\hline M 13 & 8.2 & 4.5 & 6.7 & 1.30 \\
\hline M 21 & 5.7 & 4.5 & 6.8 & 0.90 \\
\hline M 22 & 7.2 & 4.4 & 6.6 & 1.14 \\
\hline M 23 & 8.4 & 4.4 & 6.6 & 1.33 \\
\hline M 31 & 5.7 & 4.4 & 6.4 & 0.90 \\
\hline M 32 & 7.2 & 4.3 & 6.4 & 1.14 \\
\hline M 33 & 8.4 & 4.3 & 6.5 & 1.33 \\
\hline M 41 & 5.1 & 4.5 & 6.7 & 0.81 \\
\hline M 42 & 6.9 & 4.2 & 6.3 & 1.10 \\
\hline M 43 & 8.0 & 4.0 & 6.0 & 1.27 \\
\hline M 51 & 4.5 & 4.1 & 6.1 & 0.71 \\
\hline M 52 & 6.5 & 4.1 & 6.1 & 1.03 \\
\hline M 53 & 7.2 & 4.1 & 6.2 & 1.14 \\
\hline
\end{tabular}

من خلال الجدول (9) يلاحظ أن مقاومة الثد الانشطاري للمزجة الخرسانية الحاوية على نسبة غبار السليكا

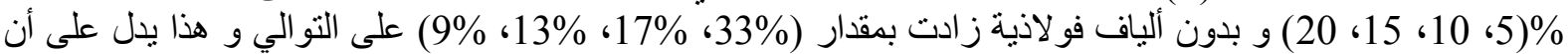

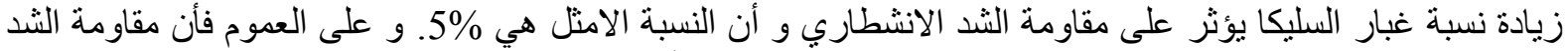

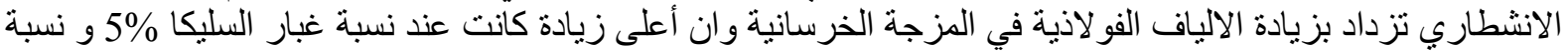

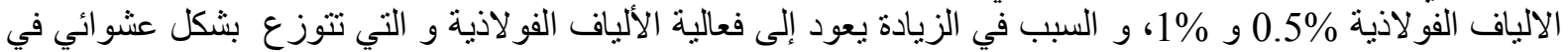

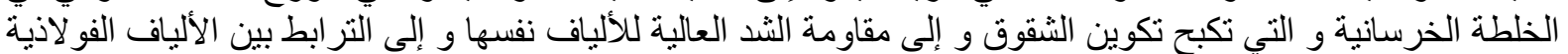

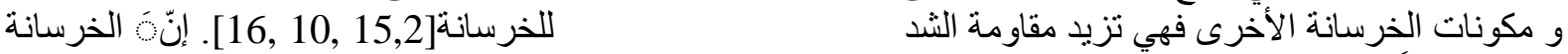

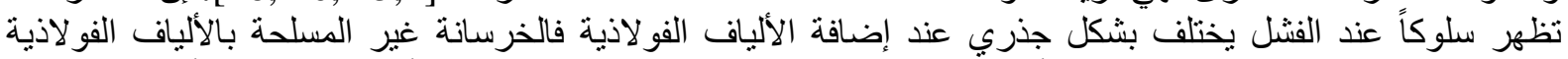

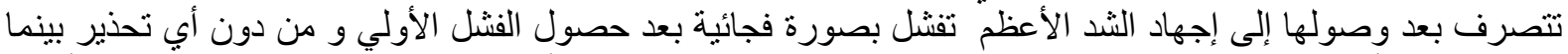

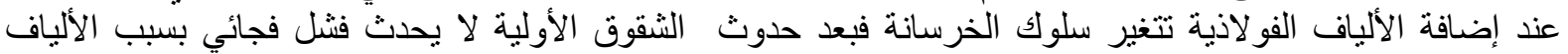

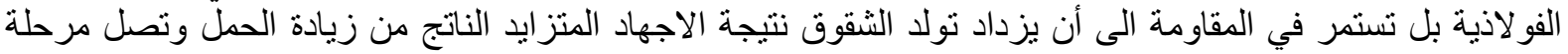

يلاحظ من الجدول (9) تقارب قيم مقاومة الثد الانشطاري للمزجة المرجعية مع القيم الناتجة من معادلة الكود

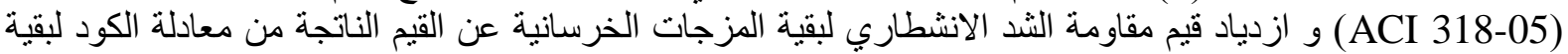
المزجات الخرسانية و السبب يعود الى فعالية غبار السليكا و الالياف الفولاذية في زياتية التيادة مقاومة الثد الانشطاري للخرسانة. و تم تمثيل العلاقة بين مقاومة الثد الانشطاري للمزجات الخرسانية و مقاومة الثند الانشطاري للمزجة المرجعية ونسب الالياف الفو لاذية ونسب غبار السليكا من خلال المعادلة الآتية:

$$
f_{s p}=f_{s p r}+83.5 v_{f}+2.6 w_{s i}
$$


يوسف: الخصائص الميكانيكية للخرسانة عالية المقاومة المسلحة بالالياف الفولاذية

الجدول (9) قيم مقاومة الثد الانشطاري

\begin{tabular}{|c|c|c|c|c|c|}
\hline Mix Designation & $\begin{array}{c}\text { Splitting Tensile Strength } f_{s p} 28 \\
\text { (days) (MPa) }\end{array}$ & $\frac{f_{s p}}{f_{s p r}}$ & $\frac{f_{s p}}{f_{c}}$ & $\frac{f_{s p}}{f_{r}}$ & $\begin{array}{r}(\mathrm{MPa}) 0.56 \sqrt{f_{c}} \\
(\mathrm{Eq} \mathrm{ACI} 318-05)\end{array}$ \\
\hline $\mathrm{M} 11=f_{s p r}$ & 3.50 & 1.00 & 0.09 & 0.56 & 3.4 \\
\hline M 12 & 3.80 & 1.09 & 0.09 & 0.51 & 3.5 \\
\hline M 13 & 4.33 & 1.24 & 0.09 & 0.53 & 3.8 \\
\hline M 21 & 4.66 & 1.33 & 0.09 & 0.86 & 3.8 \\
\hline M 22 & 4.96 & 1.42 & 0.11 & 0.69 & 3.8 \\
\hline M 23 & 4.96 & 1.42 & 0.11 & 0.59 & 3.7 \\
\hline M 31 & 4.11 & 1.17 & 0.09 & 0.72 & 3.6 \\
\hline M 32 & 4.25 & 1.21 & 0.10 & 0.60 & 3.6 \\
\hline M 33 & 4.25 & 1.21 & 0.09 & 0.51 & 3.7 \\
\hline M 41 & 3.97 & 1.13 & 0.09 & 0.79 & 3.8 \\
\hline M 42 & 4.11 & 1.17 & 0.10 & 0.61 & 3.6 \\
\hline M 43 & 4.25 & 1.21 & 0.11 & 0.54 & 3.4 \\
\hline M 51 & 3.8 & 1.09 & 0.10 & 0.84 & 3.4 \\
\hline M 52 & 4.46 & 1.27 & 0.12 & 0.69 & 3.5 \\
\hline M 53 & 4.82 & 1.38 & 0.12 & 0.67 & 3.5 \\
\hline
\end{tabular}

\section{4-3-3: منحني الإجهاد و الانفعال}

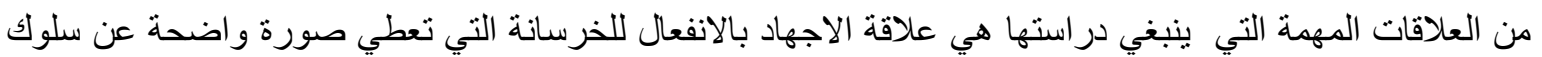

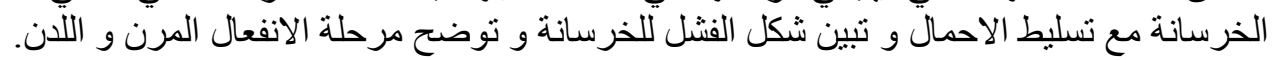
يتبين من الاشكال (3) و (4) و (5) و الجدول (10) ارتفاع مرحلة الاجهاد العظمى للخرسانة بإضافة غبار

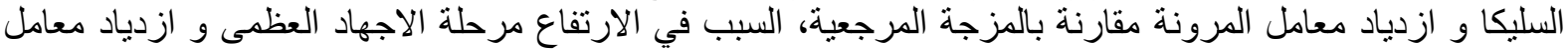

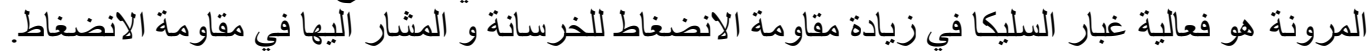

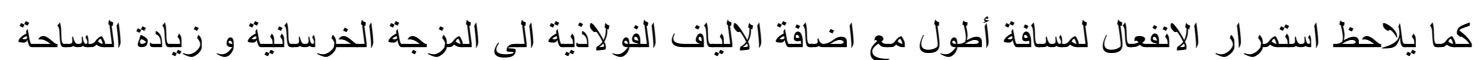

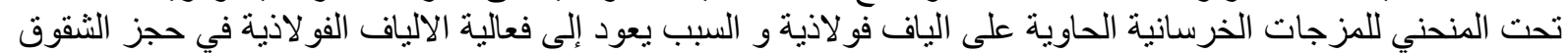

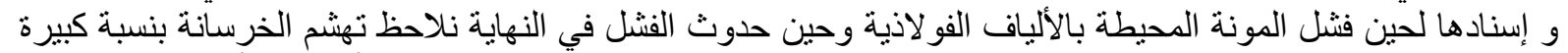

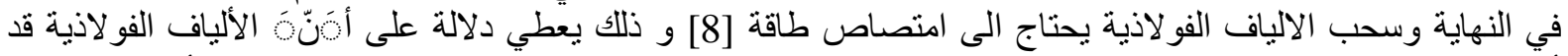

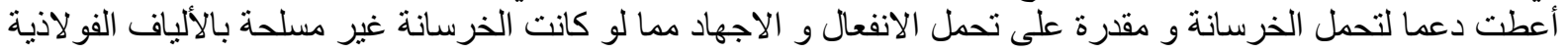

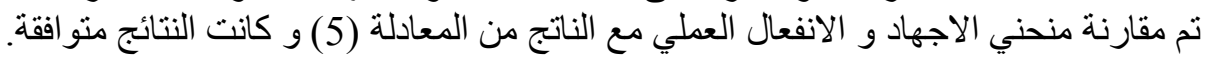
تم تمثيل منحني الإجهاد و الانفعال بمعادلة تجريبية استخرجت بتحليل البيانات العملية:

$$
y=\frac{(\alpha x)}{\left(\alpha-1+x^{b}\right)}
$$

$$
\begin{aligned}
& \text { حيث أن قيم كل من (ba , ) تستخرج من المعادلتين الآتيتين: } \\
& a=2.257+6.435 w_{a i}+40.048 v_{f}-1014.339 w_{g i} v_{f} \\
& b=1.988+8.484 w_{a i}+37.955 v_{f}-1236.972 w_{a i} v_{f}
\end{aligned}
$$




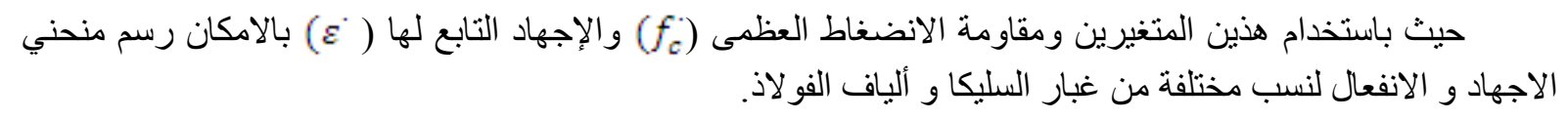

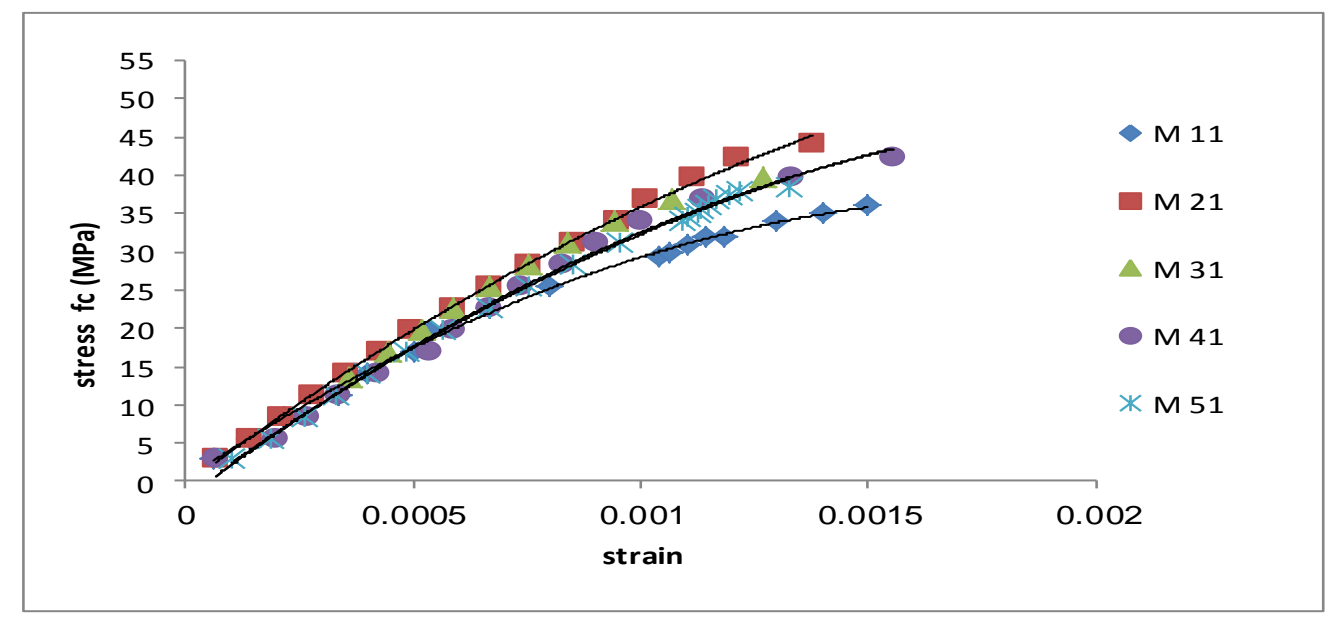

الشكل (3) تأثير غبار السليكا على منحني الاجهاد و الانفعال (بدون الياف فولاذية)

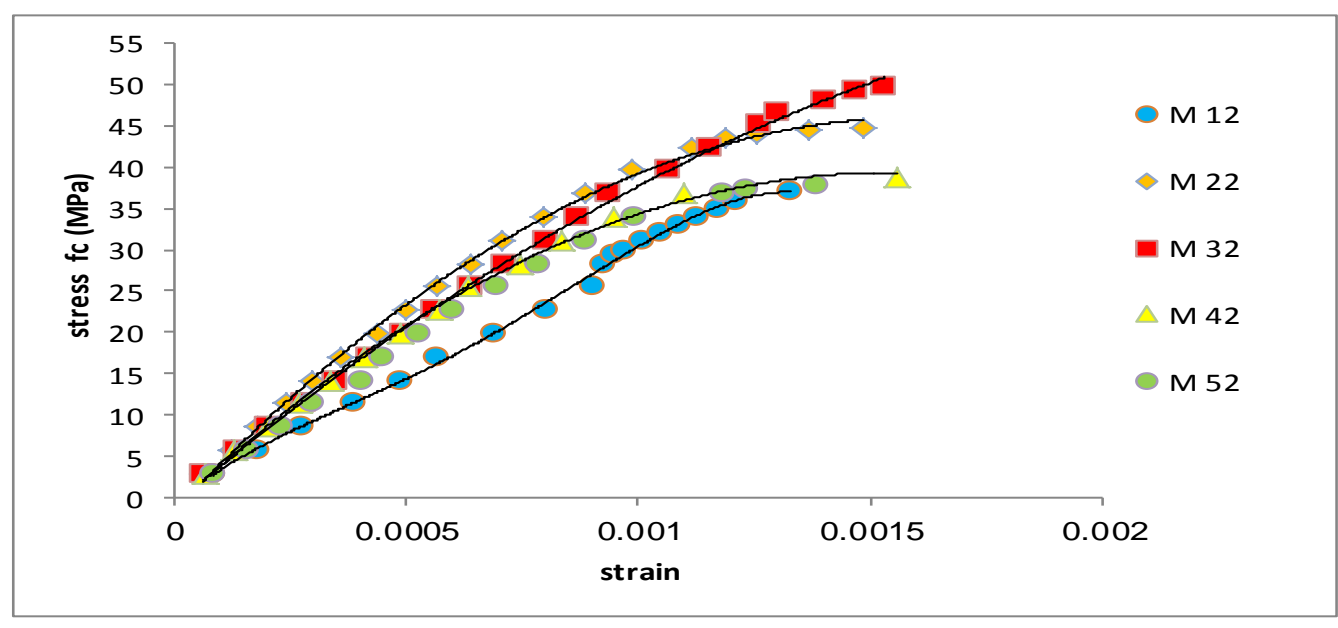

الثكل (4) تأثير غبار السليكا على منحني الاجهاد و الانفعال (نسبة الالياف الفولانية 0.5\%)

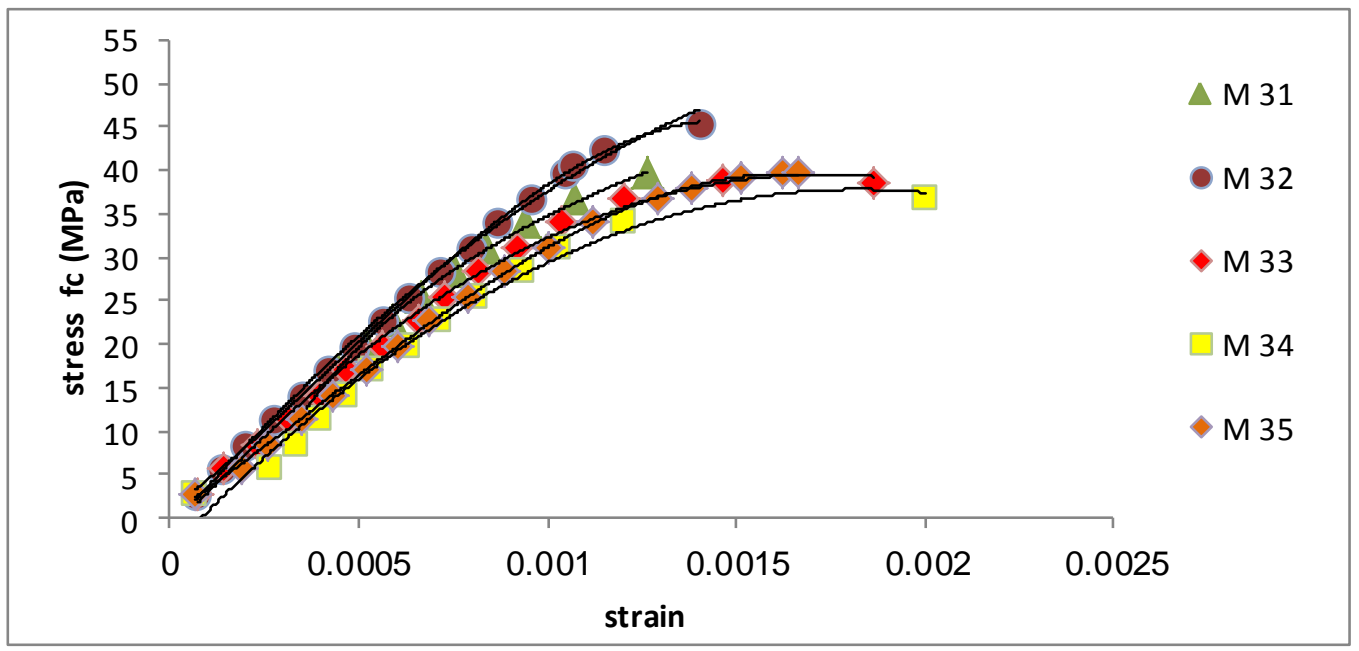

الثكل (5) تأثثر غبار السليكا على منحني الاجهاد و الانفعال (نسبة الالياف الفولانية 1\%) 
يوسف: الخصائص الميكاتيكية للخرسانة عالية المقاومة المسلحة بالالياف الفولانية

الجدول (10) الاجهاد و الانفعال الاقصى للمزجات الخرسانية

\begin{tabular}{|c|c|c|c|}
\hline Designation Mix & $\begin{array}{c}f_{c}(\text { MPa }) \\
(\mathbf{2 8 ~ d a y s})\end{array}$ & strain at failure & $\left(\boldsymbol{f}_{\boldsymbol{c}} / \boldsymbol{f}_{\text {cr }}\right)$ \\
\hline M 11=( $\left.\boldsymbol{f}_{\text {cr }}\right)$ & 36.70 & 0.0015000 & 1.00 \\
\hline M 12 & 39.16 & 0.0012467 & 1.07 \\
\hline M 3 & 45.70 & 0.0014333 & 1.25 \\
\hline M 1 & 47.10 & 0.0015333 & 1.28 \\
\hline M 22 & 45.10 & 0.0014867 & 1.23 \\
\hline M 23 & 44.50 & 0.0014000 & 1.21 \\
\hline M 32 & 42.00 & 0.0012667 & 1.14 \\
\hline M 33 & 41.80 & 0.0015333 & 1.14 \\
\hline M 41 & 43.20 & 0.0014667 & 1.17 \\
\hline M 42 & 46.40 & 0.0015600 & 1.26 \\
\hline M 43 & 40.60 & 0.0015600 & 1.10 \\
\hline M 51 & 37.90 & 0.0020000 & 1.03 \\
\hline M 52 & 37.80 & 0.0013267 & 1.03 \\
\hline M 53 & 38.50 & 0.0013867 & 1.05 \\
\hline
\end{tabular}

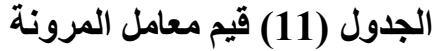

\begin{tabular}{|c|c|c|}
\hline $\begin{array}{c}\text { Designation } \\
\text { Mix }\end{array}$ & $\begin{array}{c}\text { Modulus of } \\
\text { elasticity (MPa) }\end{array}$ & $\left(\boldsymbol{E}_{\boldsymbol{c}} / \boldsymbol{E}_{\boldsymbol{c r}}\right)$ \\
\hline M 11= $\left(\boldsymbol{E}_{\boldsymbol{c r}}\right)$ & 31914 & 1.00 \\
\hline M 12 & 33883 & 1.06 \\
\hline M 13 & 36825 & 1.15 \\
\hline M 21 & 39875 & 1.25 \\
\hline M 22 & 40146 & 1.26 \\
\hline M 23 & 40474 & 1.27 \\
\hline M 31 & 38106 & 1.19 \\
\hline M 32 & 37733 & 1.18 \\
\hline M 33 & 39722 & 1.25 \\
\hline M 41 & 36553 & 1.15 \\
\hline M 42 & 36600 & 1.15 \\
\hline M 43 & 36487 & 1.14 \\
\hline M 51 & 36000 & 1.13 \\
\hline M 52 & 37900 & 1.19 \\
\hline M 53 & 39737 & 1.25 \\
\hline
\end{tabular}

معامل المرونة: يعبر عن العلاقة بين الاجهاد

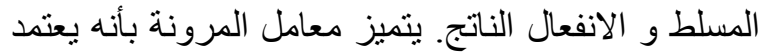

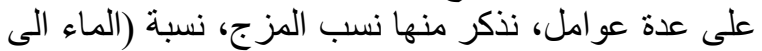
المواد الاسمنتية)، مقاومة الخرسانة، كثانة كنافة الخرسانة،

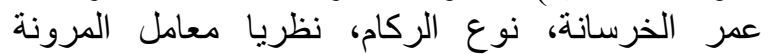

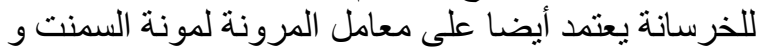

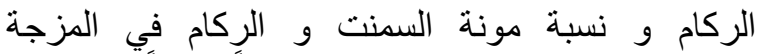

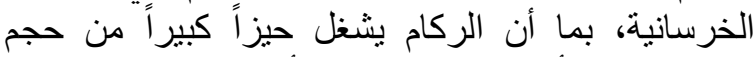

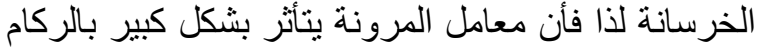

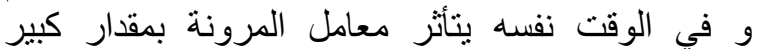

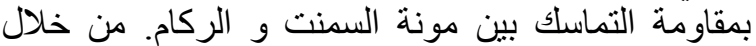

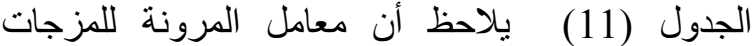

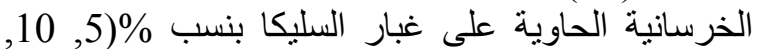
15, 20) و بدون ألياف فولاذية قد ازداد بنسب \%

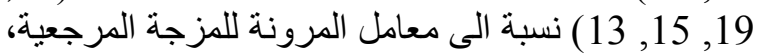

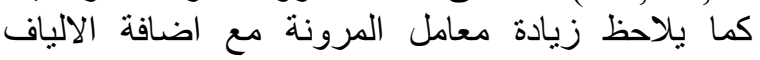

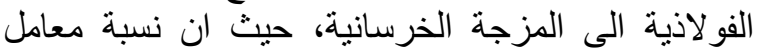
المرونة للمزجات الخرسانية الحاوية على غلى غبار السليكا بنسب \% \%, 5, 10, 15, 20) و الحاوية على الكية ألياف

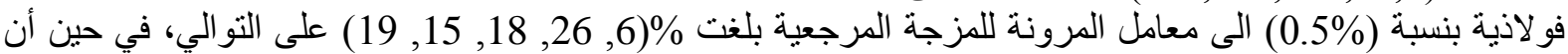
تللك النسب بلغت \% \%(15, 27, 25, 14, 25) للخرسانة الحاوية على ألياف فو لاذية بنسبة (1\% (15).

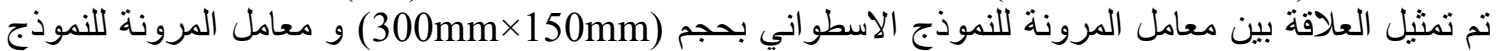
الاسطو اني بحجم (300mm×150mm) للمزجة المرجعية و نسب الالياف الفولاذية و نسب غبار السليكا في المعادلة الآتية:

$$
E_{C}=E_{C r}+464271 V_{f}+27719 w_{a i}
$$




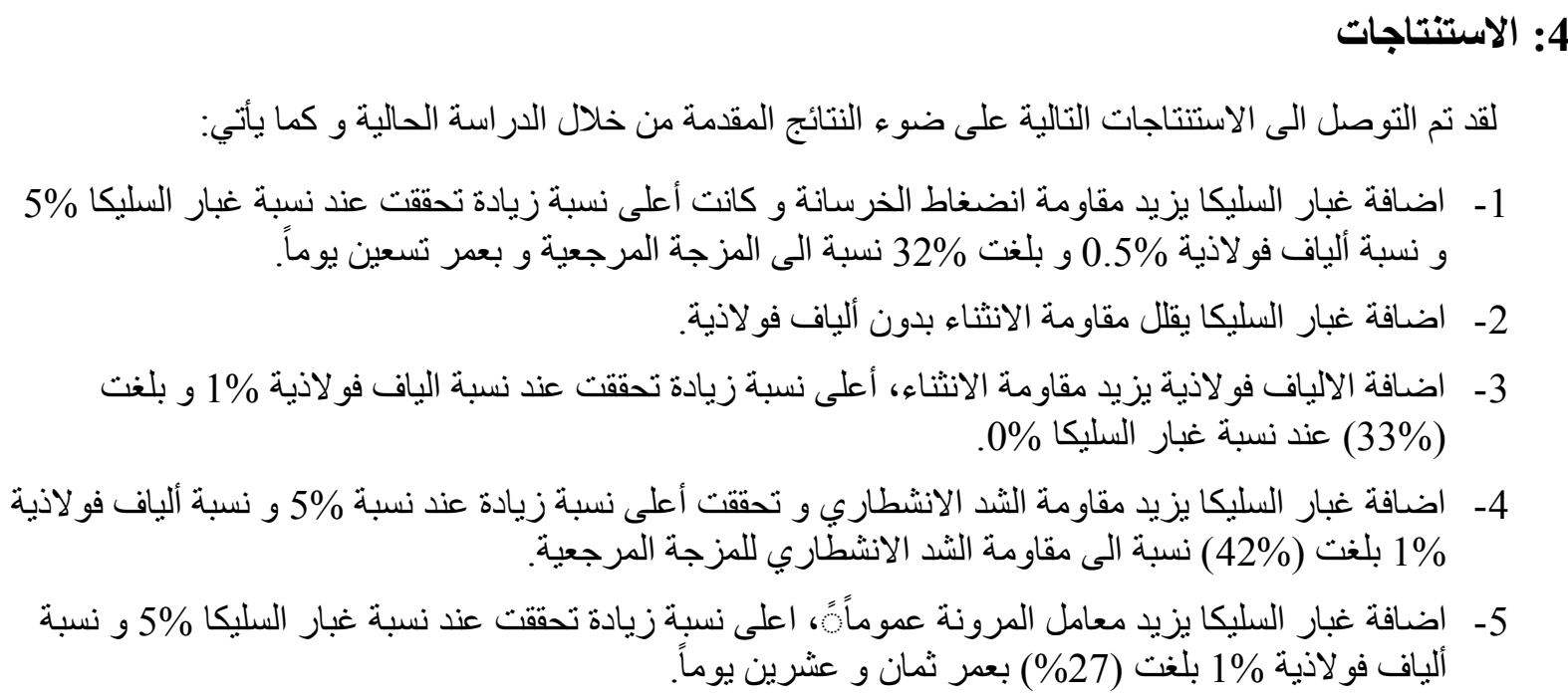

المصادر

1- ACI Committee 363 (2010) "Report on High-Strength Concrete" (ACI 363R-10), American Concrete Institute.

2- Samman, T. A. , Wafa, F. F. , Radain, T. A. "Mechanical Properties of Normal and HighStrength Concrete with Steel Fibers" JKAU:Eng.Sci.,vol.12,no.1,pp. 87-104 (1420 A.H./ 1999 A.D.).

$$
\begin{aligned}
& \text { 3- صالح, شاكر أحمد و رجب, سعيد خلف و نجم, خالد بنال "تأثثر الألياف الحديدية على الخواص الميكانيكية للخرسانة } \\
& \text { العالية الاداء" مجلة الر افدين, العدد 13, الموضوع 4, (2005). }
\end{aligned}
$$

4- Katkhuda, H., Hanayneh, B. And Shatarat, N. "Influence of Silica Fume on High Strength Lightweight Concrete", World Academy of Science, Engineering and Technology 5-8-2009, pp.781-788.

5- Sarsam, K. \& Azzawi, Z. M. "Mechanical Properties of High-Strength Fiber Reinforced Concrete" Eng. \& Tech. Journal, Vol.28, N0, 12, 2010.

6- ACI Committee 211 (2008) "Guide for Selecting Proportions For High-Strength Concrete Using Portland Cement and Other Cementitious Materials"

American Concrete Institute.

(ACI 211.4R-08),

7- Hariharan, A.R., Santhi, A.S. \& Ganesh, G.M. "Study on Strength Development of High Strength Concrete Containing Fly ash and Silica Fume" International Journal of Engineering Science and Technology (IJEST), Apr 2011, Vol.3 No. 4.

8- Pawade, P.Y., Pande, A.M. \& Nagarnaik, P.B. "Effect of Steel Fibers on Modulus of Elasticity of Concrete" International Journal of Advanced Engineering Sciences and Technologies (IJAEST), 2003 Vol 12 No.7, Issue No.2, 169-177.

9- ACI Committee E-701 (2001) "Cementitious Materials For Concrete", (ACI Education Bulletin E3-01), American Concrete Institute.

10- ACI Committee 234 (2000) "Guide for the Use of Silica Fume in Concrete"(ACI 234R96), American Concrete Institute.

11- Ashour, S.A. \& Wafa, F.F. "Effect of Steel Fibers on The Flexural Behavior of HighStrength Concrete Beams with Low Flexural Reinforcement" The Fourth Engineering Conference, Nov., 1995, Volume 2. 
12- Parrott, L. J., "The Properties of High Strength Concrete" Technical Report No. 42.417, 1969, Cement and Concrete Association, Wexham Springs, pp. 12

13- Dewar, J. D., "The Indirect Tensile Strength of Concretes of High Compressive Strength" Technical Report No. 42.377, Cement and Concrete Association, Wexham Springs, 1964, Mar., pp. 12.

14- Gao, J. W. \& Morino, K. "Mechanical Properties of Steel Fiber-reinforced, Highstrength, Lightweight Concrete" cement and concrete composites 19(1997), pp.307-313.

15- Zhang, M. H. , Li L. \& Paramasivam, P. "Shrinkage of high-strength Lightweight Aggregate Concrete Exposed to Dry Environment " ACI Materials Journal, March-April 2005,pp.86-92.

16- Zia, P., Ahmad, S., and Leming, M., "High-Performance Concretes: A State-of-Art Report (1989-1994)," FHWA-RD-97-030, 1997, Federal Highway Administration.

تم اجراء البحث في كلية ألهندسة = جامعة ألموصل 